\title{
Epidemiological, molecular characterization and antibiotic resistance of Salmonella enterica serovars isolated from chicken farms in Egypt
}

Hanem El-Sharkawy ${ }^{1,3}$, Amin Tahoun 1,3, Abd El-Galiel A. El-Gohary ${ }^{3}$, Moshira El-Abasy ${ }^{3}$, Fares El-Khayat ${ }^{3}$, Trudi Gillespie ${ }^{4}$, Yukio Kitade ${ }^{1}$, Hafez M. Hafez ${ }^{5}$, Heinrich Neubauer ${ }^{2}$ and Hosny El-Adawy ${ }^{2,3^{*}}$ [D

\begin{abstract}
Background: Salmonella is one of major causes of foodborne outbreaks globally. This study was conducted to estimate the prevalence, typing and antibiotic susceptibilities of Salmonella enterica serovars isolated from 41 broiler chicken farms located in Kafr El-Sheikh Province in Northern Egypt during 2014-2015. The clinical signs and mortalities were observed.

Results: In total 615 clinical samples were collected from broiler flocks from different organs (liver, intestinal content and gall bladder). Salmonella infection was identified in 17 (41\%) broiler chicken flocks and 67 Salmonella isolates were collected. Recovered isolates were serotyped as 58 (86.6\%) S. enterica serovar Typhimurium, 6 (9\%) S. enterica serovar Enteritidis and 3 (4.5\%) were non-typable. The significant high mortality rate was observed only in 1-week-old chicks. sopE gene was detected in $92.5 \%$ of the isolates which indicating their ability to infect humans. All S. enterica serovar Enteritidis isolates were susceptible to all tested antimicrobials. The phenotypically resistant S. enterica serovar Typhimurium isolates against ampicillin, tetracycline, sulphamethoxazole and chloramphenicol were harbouring BlaTEM, (tetA and tetC), (sul1 and sul3) and (cat1 and floR), respectively. The sensitivity rate of S. enterica serovar Typhimurium to gentamycin, trimethoprim/sulphamethoxazole and streptomycin were 100, 94.8, 89.7\%, respectively. The silent streptomycin antimicrobial cassettes were detected in all Salmonella serovars. A class one integron (dfrA12, orfF and aadA2) was identified in three of S. enterica serovar Typhimurium strains.
\end{abstract}

Conclusions: To the best of our knowledge, this study considered first report discussing the prevalence, genotyping, antibiotic susceptibility and public health significance of S. enterica serovars in broilers farms of different ages in Delta Egypt. Further studies are mandatory to verify the location of some resistance genes that are within or associated with the class one integron.

Keywords: Salmonella, Broiler, Epidemiology, Antimicrobial, Integron

\section{Background}

In spite of significant improvement in technology and hygienic practices at all stages of poultry production accompanied with advanced improvement in

\footnotetext{
*Correspondence: hosny.eladawy@fli.de

${ }^{2}$ Institute of Bacterial Infections and Zoonoses, Friedrich-Loeffler-Institut, Naumburger Str. 96a, 07743 Jena, Germany

Full list of author information is available at the end of the article
}

public sanitation, salmonellosis and Salmonella infections remains a persistent threat to human and animal health. In many countries high incidence of salmonellosis in man appears to be caused by infection derived from contaminated eggs, poultry meat and meat-products. The contaminated products cause disease as a result of inadequate cooking or cross contamination of working surfaces in kitchen environment [1-3]. 
The genus Salmonella of the family Enterobacteriaceae includes more than 3000 distinct serovars that have many host species and cause different diseases; most of which show little specificity for their host species [4-7]. The genus Salmonella can roughly be classified into three categories or groups: Group 1, highly host-adapted and invasive serovars: this group includes species restricted and invasive Salmonella such as S. Pullorum, S. Gallinarum in poultry and $S$. Typhi in humans. Group 2, non-host-adapted and invasive serovars: this group consists of approximately 10-20 serovars that are able to cause an invasive infection in poultry and may be capable of infecting humans. Currently, the most important serovars are $S$. Enteritidis, $S$. Typhimurium, $S$. Hadar, $S$. Heidelberg, $S$. Saintpaul and $S$. Infantis. Group 3, nonhost-adapted and non-invasive serovars: most serovars of the genus Salmonella belong to this group and may cause disease in humans and other animals [8-14].

Although the acute enteritis caused by Salmonella species in humans is usually self-limiting, salmonellosis may be complicated especially in younger and older ages by severe systemic sequelae depending on serotype and on host-specific factors [15-17].

Salmonella enterica serovar Typhimurium and S. enterica serovar Enteritidis have been identified as the predominant serotypes present in Egyptian poultry farms [18].

Salmonella enterica serovar Enteritidis has been associated with disease in broiler breeding stock and can be transmitted vertically to their progeny [19]. Infection of adult chickens with $S$. enterica serovar Typhimurium is usually without clinical manifestation [20]. S. enterica serovar Enteritidis can inhabit the intestinal tract of several bird species such as chickens, turkeys and game birds and has the ability to survive outside of the host for over 1 year. S. enterica serovar Enteritidis infection in adult poultry is usually asymptomatic and infected bird will become a chronic carrier [21, 22]. In chickens up to 6 weeks of age $S$. enterica serovar Enteritidis may produce clinical symptoms including depression, disinclination to move, and diarrhoea, with high mortality especially in chicks less than 1 week of age [23], while older chicks may show uneven growth and stunting. Laying hens sometimes produce S. enterica serovar Enteritidis contaminated eggs leading to public health concerns [19]. The diseased birds may show lesions of pericarditis, perihepatitis and septicaemia. The mortality and morbidity vary and has been found to depend upon the dosage and phage type of the S. enterica serovar Enteritidis infection $[24,25]$.

Antimicrobial resistance is increasingly becoming an issue with salmonellosis infections in both animals and humans [26]. Understanding the key mechanisms involved in the evolution of antibiotics resistance in bacteria may aid scientific innovations aimed at controlling antimicrobial resistance [27, 28]. Bacteria can acquire resistance genes through mobile elements such as plasmids, which provide flexibility to host bacteria and help in the spread and distribution of these genes across diverse bacterial populations [29].

The inappropriate use of antibiotics in chicken farms in developing countries, including Egypt, is thought to be one of the main reasons for the increase in multidrug resistant bacteria [30]. These multidrug resistant bacteria including both $S$. enterica serovar Typhimurium, and S. enterica serovar Enteritidis that have the potential to infect humans and with a consequent failure of treatment can lead to systemic infection and death [31].

In this study, the incidence and antimicrobial resistance of S. enterica serovars Typhimurium and Enteritidis isolated from broiler chicken farms in Kafr El-Sheikh Province, Northern Egypt was reported. Determination of genes associated with antimicrobial resistance was investigated by examining the distribution of mobile integrons that carry the multidrug resistance cassettes within the genome of the isolated strains.

\section{Methods}

\section{Sampling strategy and Salmonella isolation}

This study was conducted in 41 broiler flocks located in Kafr El-Sheikh Province in Delta Egypt. Twenty flocks of 1 -week-old birds and 21 flocks of 5-week-old birds were investigated. The observed clinical symptoms were observed and recorded (Table 1). Five living morbid birds from each flock were randomly selected and humanly sacrificed. At necropsy, sections of liver and intestinal wall plus contents were collected aseptically and processed for Salmonella isolation. From the same bird bile was aspirated from the gall bladder. Wetted cotton swabs in bacteriological transport media were used to collect samples from each specimen. Collected swabs and tissue samples were immediately frozen on ice and stored at $-20{ }^{\circ} \mathrm{C}$ for further investigation within $5 \mathrm{~h}$. Each tissue sample and swabs were inoculated in $10 \mathrm{ml}$ selenite $\mathrm{F}$ broth (Oxoid, UK) and incubated at $37{ }^{\circ} \mathrm{C}$ overnight. A loopful of inoculated broth was streaked on selective Salmonella Shigella (SS) agar (Oxoid, UK) and incubated at $37{ }^{\circ} \mathrm{C}$ overnight. The suspected colony was sub-cultured on Xylose lysine deoxycholate (XLD) agar (Oxoid, UK) and on brilliant green (BG) agar (Oxoid, UK) and incubated at $37^{\circ} \mathrm{C}$ for 16-18 $\mathrm{h}$. The suspected colonies were collected for further biochemical identification using API 20E (BioMérieux, Marcy-l'Étoile, France). 
Table 1 Flock description, signs, mortalities and Salmonella isolation rate from broiler chicken farms in the Kafr ElSheikh Province Northern Egypt

\begin{tabular}{|c|c|c|c|c|c|}
\hline Flock no. & $\begin{array}{l}\text { No. } \\
\text { of birds }\end{array}$ & Age/day & Clinical signs & Mortality, n (\%) & Isolation results \\
\hline 1 & 10,000 & 1 & Pasty diarrhea, blindness, lameness and high mortality & $850(8.5)$ & S. Enteritidis \\
\hline 2 & 10,000 & 2 & Inappetence and respiratory manifestation & $110(1.1)$ & Negative \\
\hline 3 & 15,000 & 3 & $\begin{array}{l}\text { Pasty diarrhea, conjunctivitis, lowering in body weight } \\
\text { and high mortalities }\end{array}$ & $975(6.5)$ & S. Enteritidis \\
\hline 4 & 15,000 & 4 & Inappetence, ruffling feather and nervous signs & $360(2.4)$ & Negative \\
\hline 5 & 25,000 & 5 & Lowering body rate and respiratory signs & $550(2.2)$ & Negative \\
\hline 6 & 2000 & 7 & $\begin{array}{l}\text { Pasty diarrhea, loss of appetite, ruffling feather and } \\
\text { high mortalities }\end{array}$ & $190(9.5)$ & S. Typhimurium \\
\hline 7 & 5000 & 7 & $\begin{array}{l}\text { Decreased body weight, diarrhea, dehydration and } \\
\text { high mortalities }\end{array}$ & $415(8.3)$ & S. Typhimurium \\
\hline 8 & 20,000 & 4 & Decreased body weight & $500(2.5)$ & Negative \\
\hline 9 & 10,000 & 6 & $\begin{array}{l}\text { Whitish diarrhea, high mortalities, and decreased } \\
\text { body weight }\end{array}$ & $1160(11.6)$ & S. Typhimurium \\
\hline 10 & 12,000 & 7 & Inappetence, diarrhea and lowering body weight & $540(4.5)$ & Negative \\
\hline 11 & 25,000 & 7 & Inability to move and nervous signs & $850(3.4)$ & Negative \\
\hline 12 & 30,000 & 5 & Diarrhea, drop in feed intake and high mortalities & $2610(8.7)$ & S. Typhimurium \\
\hline 13 & 5000 & 4 & Respiratory signs and decreased body weight & $225(4.5)$ & Negative \\
\hline 14 & 30,000 & 3 & Inappetence, lowering growth rate & $840(2.8)$ & Negative \\
\hline 15 & 15,000 & 6 & $\begin{array}{l}\text { Whitish diarrhea, conjunctivitis and decreased body } \\
\text { weight }\end{array}$ & $945(6.3)$ & S. Typhimurium \\
\hline 16 & 10,000 & 4 & $\begin{array}{l}\text { Diarrhea and decrease in body weight and respiratory } \\
\text { signs }\end{array}$ & $350(3.5)$ & Negative \\
\hline 17 & 10,000 & 5 & Inappetence, mortalities, lameness and diarrhea & $550(5.5)$ & S. Typhimurium \\
\hline 18 & 20,000 & 6 & Decreased body weight and respiratory signs & $640(3.2)$ & Negative \\
\hline 19 & 12,000 & 5 & Diarrhea, blindness and high mortality & $648(5.4)$ & S. Typhimurium \\
\hline 20 & 10,000 & 5 & Respiratory and nervous signs & $420(4.2)$ & Negative \\
\hline 21 & 15,000 & 33 & Inappetence and respiratory manifestation & $375(2.5)$ & S. Typhimurium \\
\hline 22 & 20,000 & 32 & Decreased body weight & $280(1.4)$ & Negative \\
\hline 23 & 30,000 & 33 & Mortalities & $660(2.2)$ & Negative \\
\hline 24 & 25,000 & 29 & Nervous signs & $625(2.5)$ & Negative \\
\hline 25 & 15,000 & 34 & Decreased body weight & $345(2.3)$ & S. Typhimurium \\
\hline 26 & 20,000 & 29 & Decreased body weight & $460(2.3)$ & Negative \\
\hline 27 & 30,000 & 30 & Decreased body weight & $780(2.6)$ & Negative \\
\hline 28 & 10,000 & 31 & Respiratory signs and high mortality & $330(3.3)$ & S. Typhimurium \\
\hline 29 & 5000 & 28 & Respiratory signs and mortalities & $165(3.3)$ & Negative \\
\hline 30 & 15,000 & 33 & Inappetence and mortalities & $480(3.2)$ & Negative \\
\hline 31 & 20,000 & 33 & Respiratory signs and high mortality & $600(3.0)$ & S. Typhimurium \\
\hline 32 & 20,000 & 32 & Inappetence and respiratory manifestation & $700(3.5)$ & S. Typhimurium \\
\hline 33 & 10,000 & 28 & Mortalities & $200(2.0)$ & Negative \\
\hline 34 & 20,000 & 29 & Mortalities & $480(2.4)$ & Non typable Salmonella (three isolates) \\
\hline 35 & 10,000 & 33 & Respiratory signs and mortalities & $290(2.9)$ & S. Typhimurium \\
\hline 36 & 5000 & 33 & Lower body weight and respiratory signs & $135(2.7)$ & Negative \\
\hline 37 & 15,000 & 32 & Nervous signs & $345(2.3)$ & Negative \\
\hline 38 & 20,000 & 33 & Inappetence and mortalities & $540(2.7)$ & Negative \\
\hline 39 & 10,000 & 31 & Respiratory signs and mortalities & $320(3.2)$ & S. Enteritidis \\
\hline 40 & 5000 & 29 & Nervous manifestations and inappetence & $140(2.8)$ & Negative \\
\hline 41 & 25,000 & 35 & Opisthosomas and ruffled feather & $825(3.3)$ & Negative \\
\hline
\end{tabular}




\section{Genomic DNA extraction and purification}

The identified bacterial cultures were cultivated on SS agar and inoculated on Luria-Bertani (LB) broth (Oxoid, UK) and incubated at $37{ }^{\circ} \mathrm{C}$ overnight. The DNA was extracted from bacterial cultures on broth using Qiagen DNA extraction kit (Qiagen, UK) according to the manufacturer's instructions.

\section{Molecular biological identification and differentiation of Salmonella serovars}

In order to make a rapid and definite diagnosis of Salmonella, PCR was conducted using primers to detect the gene marker for S. enterica invA [32], sdfl primers specific for detection of S. enterica serovars Enteritidis [33], and Typh, Sal and $f l i C$ specific primers for serovar $S$. Typhimurium [34, 35] (Table 2).

invA positive strains were tested for the presence of the sefA gene, which encodes for SEF14 fimbriae that can be detected in S. enterica serovar Enteritidis strains and will also be present in the poultry-associated serotype $S$. Gallinarum.

In order to detect the zoonotic potential of our isolated strains of S. enterica serovar Enteritidis and S. enterica serovar Typhimurium we screened for the presence of the sopE gene [36].

The PCR reaction was geared to a previously described protocol for Salmonella [32-36]. Conserved forward and reverse primers (Eurofins, Japan) were used to generate the target amplicon (Table 1). The PCR cycling conditions were carried out as the following: initial denaturation at $94{ }^{\circ} \mathrm{C}$ for $5 \mathrm{~min}$. Thirty cycles of amplification were run for $5 \mathrm{~s}$, at $94{ }^{\circ} \mathrm{C}, 10 \mathrm{~s}$ at $68^{\circ} \mathrm{C}$ and $20 \mathrm{~s}$ at $72{ }^{\circ} \mathrm{C}$, with the final extension continuing at $72{ }^{\circ} \mathrm{C}$ for $7 \mathrm{~min}$. Different annealing temperatures were used as described in Table 1. Five microliter aliquots of reaction mixture were electrophoresed through $1.5 \%$ agarose gels (Nippongene, Japan).

\section{Determination and sequencing of class 1 integrons}

The class one integrons PCR fragments were purified from the agarose gel using Nucleospin Gel Extraction Kit (Macherey-Nagel, Germany) and sequenced (Genome centre-Gifu University, Japan). The sequencing results were analysed using BLAST webpage (http://blast.ncbi. nlm.nih.gov/Blast.cgi).

\section{Antimicrobial susceptibility testing}

The antimicrobial susceptibility testing was performed using the Kirby-Bauer disc diffusion test [37] at the Clinical Veterinary Microbiology Laboratory of the Royal Dick School of Veterinary Study, University of Edinburgh. Briefly, one colony from the SS agar plate of each strain was picked up and streaked onto MuellerHinton blood agar (Oxoid, UK) and incubated at $37{ }^{\circ} \mathrm{C}$ overnight. Bacterial colonies were suspended in $0.9 \%$ $\mathrm{NaCl}$ to obtain a McFarland turbidity of 0.5 (Dr. Lange, photometer CADAS 30, Berlin, Germany) that containing about $1-2 \times 10^{8}$ colony forming units $(\mathrm{CFU}) / \mathrm{ml}$ of Escherichia coli strain American Type Culture Collection (ATCC) 25922. Approximately, $300 \mu$ of the saline suspension was spread onto the surface of a Mueller-Hinton agar plate (Oxoid, UK) using a sterile swab.

Table 2 Primer sequences and their corresponding genes used for the detection of S. enterica serovar Enteritidis and S. enterica serovar Typhimurium

\begin{tabular}{|c|c|c|c|c|}
\hline Gene & Oligonucleotide sequence $\left(5^{\prime}-3^{\prime}\right)$ & Annealing $\left({ }^{\circ} \mathrm{C}\right)$ & Amplicon size (bp) & Reference \\
\hline invA-F & GCT GCG CGC GAA CGG CGA AG & 62 & 389 & [32] \\
\hline invA-R & TCC CGG CAG AGT TCC CATT & & & \\
\hline$S d f l-F$ & TGTGTTTTATCTGATGCAAGAGG & 58 & 293 & [33] \\
\hline$S d f-R$ & CGTTCTTCTGGTACTTACGATGAC & & & \\
\hline Sdfll-F & GCGAATATCATTCAGGATAAC & 58 & 450 & [33] \\
\hline$S d f l-R$ & GCATGTCATACCGTTGTGGA & & & \\
\hline Sdflll-F & GCTGACTCACACAGGAAATCG & 58 & 350 & [33] \\
\hline Sdflll-R & TCTGATAAGACTGGGTTTCACT & & & \\
\hline SefA-F & GCC GTA CAC GAG CTT ATA GA & 55 & 250 & [33] \\
\hline SefA-R & ACC TAC AGG GGC ACA ATA AC & & & \\
\hline Sal flic-F & CCCCGCTTACAGGTGGACTAC & 62 & 433 & [35] \\
\hline Sal flic-R & AGCGGGTTTTCGGTGGTTGT & & & \\
\hline SopE-F & ACA CAC TTT CCA CGA GGA AGC G & 55 & 398 & {$[36]$} \\
\hline SopE-R & GGA TGC CTT CTG ATG TTG ACT GG & & & \\
\hline Typh-F & TTGTTCACTTTTTACCCCTGA A & 55 & 401 & [34] \\
\hline Typh-R & CCCTGACAGCCGTTAGATATT & & & \\
\hline
\end{tabular}


The antimicrobial discs (Oxoid, UK) of six clinically used antibiotics that are used in the Egyptian poultry production (tetracycline $30 \mu \mathrm{g}$, ampicillin $10 \mu \mathrm{g}$, sulfamethoxazole/trimethoprim $25 \mu \mathrm{g}$, gentamicin $10 \mu \mathrm{g}$, streptomycin $25 \mu \mathrm{g}$ and chloramphenicol $30 \mu \mathrm{g}$ ) were distributed onto the surface of the Mueller-Hinton agar plates using a Multi-disc dispenser (Oxoid, UK). The plates were incubated at $37{ }^{\circ} \mathrm{C}$ overnight. The diameters of the inhibited zones were measured using sliding callipers and interpreted using standard break points according to the method described by The European Committee on Antimicrobial Susceptibility Testing [38] (Table 3).

The gene associated with antibiotic resistance was tested in isolated Salmonella strains. Isolates were screened for the presence of 18 genes known to be associated with resistance to the seven tested antibiotics (Table 4).

\section{Statistical analysis}

The mortality rate associated with Salmonella infection and the rate of $S$. enterica serovar Typhimurium isolation from internal organs were analysed by the student $t$ test [39].

\section{Results}

Clinical signs, mortality and incidence of Salmonella isolation from broiler flocks

Clinical symptoms of Salmonella infection observed in the 1-week-old broiler chicks included pasty diarrhea, inappetence, dehydration, growth retardation, blindness and lameness. The main gross lesions were hepatomegaly with necrotic foci, splenomegaly, pericarditis, panophthalmitis, and arthritis (Table 1).

In total 615 samples collected from intestine, liver and gall bladder from 41 broiler flocks, 67 (10.9\%) Salmonella strains were isolated. In all, $45 \%$ of the sampled 1-week-old broiler flocks (9/20) and 38\% of the screened 5-week-old broiler flocks (8/21) tested positive for Salmonella (Table 1).

The mean mortality rate $(5.23 \% \pm 2.85)$ of the 1 -weekold flocks was significantly higher $(\mathrm{P}<0.01)$ than the mean mortality rate $(2.68 \% \pm 0.52)$ in the 5 -week-old flocks. When grouped by Salmonella infection status, the mortality rate observed in the 1-week-old birds was significantly higher $(\mathrm{P}<0.001)$ in the Salmonella positive flocks $(7.8 \% \pm 2.07)$ compared to negative flocks $(3.1 \% \pm 0.45)$ (Table 1$)$. While, there was no significant difference in mortality rate between the infected and non-infected 5-week-old flocks ( $\mathrm{P}=0.15$, Table 1$)$.

\section{Molecular biological identification of Salmonella serovars and public health significance}

Both $S$. Enteritidis and $S$. Typhimurium serovars were isolated and identified from both the 1- and 5-week old sacrificed chicks (Table 5). Three of the collected 67 isolates were Salmonella positive but un-typable serovars.

All 67 recovered isolates were harboured invA gene (Fig. 1a). Out of 67 invA positive Salmonella strains, 6 (9.0\%) strains were positive for sefA, sdfl, sdfII and sdfIII genes (Fig. 1a) indicating S. enterica serovar Enteritidis and $58(86.6 \%)$ strains were positive for Typh, sdfII and fliC marker (Fig. 1a) indicating S. enterica serovar Typhimurium. Three Salmonella strains $(4.47 \%)$ were untypable and were positive for invA and sdfII (Fig. 1a) (Table 5).

The isolation rate of Salmonella serovars from different organs were demonstrated in Table 5. Briefly, there was highly significant difference $(\mathrm{P}<0.001)$ of $S$. enterica serovars Typhimurium isolated from the gallbladder (14.63\%) and liver $(9.76 \%)(\mathrm{P}<0.05)$ compared to those isolated from the intestine (3.9\%) (Table 5). There was no significance difference $(\mathrm{P}=0.28)$ between the isolation rate of $S$. enterica serovars Enteritidis form liver, intestine and gallbladder. The three un-typable serovars were found only at one farm and were isolated only from the intestinal samples (Table 5).

Table 3 Breakpoint values of each antimicrobial agent and phenotypic antimicrobial susceptibility profiles of 67 tested isolates used in this study according to EUCAST, 2015

\begin{tabular}{|c|c|c|c|c|c|c|c|c|c|c|c|c|c|}
\hline \multirow[t]{2}{*}{ Antimicrobial agents } & \multirow[t]{2}{*}{ Conc. $(\mu \mathrm{g})$} & \multicolumn{3}{|c|}{$\begin{array}{l}\text { Diameter of inhi- } \\
\text { bition zone }(\mathrm{mm})\end{array}$} & \multicolumn{3}{|c|}{$\begin{array}{l}\text { S. Typhimurium } \\
\text { (58) }\end{array}$} & \multicolumn{3}{|c|}{$\begin{array}{l}\text { S. Enteritidis } \\
\text { (6) }\end{array}$} & \multicolumn{3}{|c|}{$\begin{array}{l}\text { Non typable } \\
\text { (3) }\end{array}$} \\
\hline & & $\mathbf{R}$ & $\mathrm{I}$ & $\mathrm{S}$ & $\mathbf{R}$ & $\mathrm{I}$ & $\mathrm{S}$ & $\bar{R}$ & 1 & $S$ & $\mathbf{R}$ & $\mathrm{I}$ & $\bar{S}$ \\
\hline Ampicillin & 10 & $\leq 13$ & $14-16$ & $\geq 17$ & $58(100 \%)$ & - & - & - & - & $6(100 \%)$ & $3(100 \%)$ & - & - \\
\hline Chloramphenicol & 30 & $\leq 12$ & $13-17$ & $\geq 18$ & $58(100 \%)$ & - & - & - & - & $6(100 \%)$ & $3(100 \%)$ & - & - \\
\hline Gentamicin & 10 & $\leq 12$ & $13-15$ & $\geq 16$ & - & - & $58(100 \%)$ & - & - & $6(100 \%)$ & - & $3(100 \%)$ & - \\
\hline Streptomycin & 25 & $\leq 11$ & $12-14$ & $\geq 15$ & - & $6(10.3 \%)$ & 52 (89.7\%) & - & - & $6(100 \%)$ & - & $3(100 \%)$ & - \\
\hline Tetracycline & 30 & $\leq 14$ & $15-18$ & $\geq 19$ & $58(100 \%)$ & - & - & - & - & $6(100 \%)$ & $3(100 \%)$ & - & - \\
\hline Trimethoprim/sulphamethoxazole & 25 & $\leq 10$ & $11-15$ & $\geq 16$ & $3(5.2 \%)$ & - & $55(94.8 \%)$ & - & - & $6(100 \%)$ & - & $3(100 \%)$ & - \\
\hline
\end{tabular}

$S$ sensitive, I intermediate, $R$ resistance 
Table 4 Primer sequences and their corresponding genes used for detection of antimicrobial resistant genes for S. enterica serovars

\begin{tabular}{|c|c|c|c|c|c|}
\hline Gene & Primer & Nucleotide sequence (5-3) & Annealing $\left({ }^{\circ} \mathrm{C}\right)$ & Amplicon size (bp) & Reference \\
\hline \multirow[t]{2}{*}{ aadA1 } & $\mathrm{F}$ & TATCAGAGGTAGTTGGCGTCAT & 54 & 484 & [62] \\
\hline & $\mathrm{R}$ & GTTCCATAGCGTTAAGGTTTCATT & & & \\
\hline \multirow[t]{2}{*}{$\operatorname{aadA2}$} & $\mathrm{F}$ & TGTTGGTTACTGTGGCCGTA & 62 & 622 & [62] \\
\hline & $\mathrm{R}$ & GATCTCGCCTTTCACAAAGC & & & \\
\hline \multirow[t]{2}{*}{ aadB } & $\mathrm{F}$ & GAGCGAAATCTGCCGCTCTGG & 61 & 319 & [62] \\
\hline & $\mathrm{R}$ & CTGTTACAACGGACTGGCCGC & & & \\
\hline \multirow[t]{2}{*}{ a acC } & $\mathrm{F}$ & GGCGCGATCAACGAATTTATCCGA & 58 & 488 & [28] \\
\hline & $\mathrm{R}$ & CCATTCGATGCCGAAGGAAACGAT & & & \\
\hline \multirow[t]{2}{*}{ blaTEM } & $\mathrm{F}$ & CATTTCCGTGTCGCCCTTAT & 55 & 793 & [62] \\
\hline & $\mathrm{R}$ & TCCATAGTTGCCTGACTCCC & & & \\
\hline \multirow[t]{2}{*}{ cat 1} & $\mathrm{~F}$ & CTT GTC GCC TTG CGT ATA AT & 53 & 508 & [27] \\
\hline & $\mathrm{R}$ & ATC CCA ATG GCA TCG TAA AG & & & \\
\hline \multirow[t]{2}{*}{ cat2 } & $\mathrm{F}$ & CCGGATTGACCTGAATACCT & 56 & 572 & [62] \\
\hline & $\mathrm{R}$ & TCACATACTGCATGATGAAC & & & \\
\hline \multirow[t]{2}{*}{$d f r l$} & $\mathrm{~F}$ & GTGAAACTATCACTAATGGTAGCT & 54 & 470 & [62] \\
\hline & $\mathrm{R}$ & ACCCTTTTGCCAGATTTGGTAACT & & & \\
\hline \multirow[t]{2}{*}{ floR } & $\mathrm{F}$ & AACCCGCCCTCTGGATCAAGTCAA & 60 & 548 & [62] \\
\hline & $\mathrm{R}$ & CAAATCACGGGCCACGCTGTATC & & & \\
\hline \multirow[t]{2}{*}{ stra } & $\mathrm{F}$ & AGCAGAGCGCGCCTTCGCTC & 59 & 684 & [62] \\
\hline & $\mathrm{R}$ & CCAAAGCCCACTTCACCGAC & & & \\
\hline \multirow[t]{2}{*}{$\operatorname{str} B$} & $\mathrm{~F}$ & ATCGTCAAGGGATTGAAACC & 49 & 509 & [63] \\
\hline & $\mathrm{R}$ & GGATCGTAGAACATATTGGC & & & \\
\hline \multirow[t]{2}{*}{ sul1 } & $\mathrm{F}$ & TCACCGAGGACTCCTTCTTC & 60 & 316 & [62] \\
\hline & $\mathrm{R}$ & AATATCGGGATAGAGCGCAG & & & \\
\hline \multirow[t]{2}{*}{ sul2 } & $\mathrm{F}$ & CGGTCCGGCATCCAGCAATCC & 64 & 441 & [62] \\
\hline & $\mathrm{R}$ & CGAGAGCCACGACCGCGCC & & & \\
\hline \multirow[t]{2}{*}{ sul3 } & $\mathrm{F}$ & GAGCAAGATTTTTGGAATCG & 51 & 799 & [63] \\
\hline & $\mathrm{R}$ & CATCTGCAGCTAACCTAGGGCTTGGA & & & \\
\hline \multirow[t]{2}{*}{ tetA } & $\mathrm{F}$ & GCTACATCCTGCTTGCCTTC & 55 & 210 & [64] \\
\hline & $\mathrm{R}$ & CATAGATCGCCGTGAAGAGG & & & \\
\hline \multirow[t]{2}{*}{ tetB } & $\mathrm{F}$ & TTGGTTAGGGGCAAGTTTTG & 53 & 659 & [64] \\
\hline & $\mathrm{R}$ & GTAATGGGCCAATAACACCG & & & \\
\hline \multirow[t]{2}{*}{ tetc } & $\mathrm{F}$ & CTTGAGAGCCTTCAACCCAG & 56 & 418 & [64] \\
\hline & $\mathrm{R}$ & ATGGTCGTCATCTACCTGCC & & & \\
\hline \multirow[t]{2}{*}{ intl } & $\mathrm{F}$ & $5^{\prime}$ GGCATCCAAGCAGCAAGC-3' & 55 & 2000 & [65] \\
\hline & $\mathrm{R}$ & AAGCAGACTTGACCTGAT & & & \\
\hline
\end{tabular}

Table 5 The rate of S. enterica serovars isolation from tissue organs collected from 41 broiler chicken flocks in Kafr ElSheikh Province in Northern Egypt

\begin{tabular}{lllll}
\hline Organs & Liver & Intestine & Gallbladder & Total \\
\hline $\begin{array}{l}\text { No. of collected samples } \\
\text { No. of isolates }\end{array}$ & 205 & 205 & 205 & 615 \\
$\quad$ S. Enteritidis & $2(0.98 \%)$ & $1(0.49 \%)$ & $3(1.46 \%)$ & $6(0.98 \%)$ \\
S. Typhimurium & $20(9.76 \%)$ & $8(3.9 \%)$ & $30(14.63 \%)$ & $58(9.43 \%)$ \\
Un-typable Salmonella & 0 & $3(1.46 \%)$ & 0 & $3(0.49 \%)$ \\
Total & $22(10.74 \%)$ & $12(5.85 \%)$ & $33(16.09 \%)$ & $67(10.9 \%)$ \\
\hline
\end{tabular}




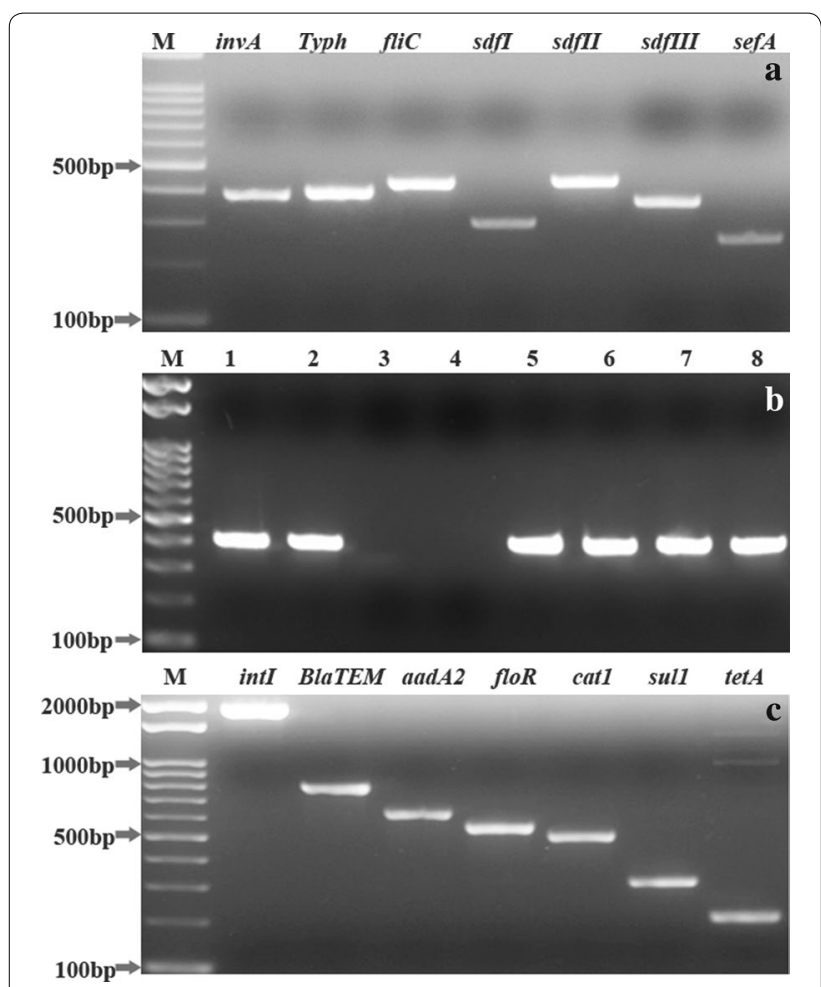

Fig. 1 a Molecular biological identification of virulent gene associated with of S. enterica serovars Enteritidis and S. enterica serovar Typhimurium. Lane M, 100 bp DNA ladder. PCR amplification of invA, Typh, fliC, sdfl, sdfll, sdflll and sefA with expected amplicon size 389, $401,433, \sim 293, \sim 450, \sim 350$ and 250 bp, respectively. b Agarose gel electrophoresis of PCR amplifications of sopE gene in S. enterica serovars Enteritidis and S. enterica serovars Typhimurium. Lane $M$, 100 bp DNA ladder. Lanes 1-2 and 5-8 were positive for the sopE with amplicon size 398 bp. c Molecular biological detection of antimicrobial resistant associated genes in S. enterica serovar Enteritidis and S. enterica serovar Typhimurium isolates. The amplified genes were intl integrons, BlaTEM, aadA2, floR, cat1, sul1, and tetA genes with amplicon size 2000, 793, 622, 548, 508, 316 and 210 bp, respectively. Lane $M, 100$ bp DNA ladder

The sop E gene was amplified in 62 (92.5\%) Salmonella isolates, indicating zoonotic and public health significance of isolated strains (Fig. 1b).

\section{Phenotypic and genotypic antimicrobial resistance}

All Salmonella serovars isolated in this study were sensitive to gentamicin. Fifty-two (89.7\%) S. enterica serovars Typhimurium isolates were susceptible to streptomycin, while six isolates $(10.3 \%)$ were intermediate. Fifty-five (94.8\%) S. enterica serovars Typhimurium isolates were sensitive to trimethoprim/sulphamethoxazole, while 3 (5.2\%) isolates were resistant. All non-typable Salmonella strains were sensitive to trimethoprim/sulphamethoxazole and streptomycin. All S. enterica serovars Typhimurium and non-typable Salmonella strains isolated were resistant to ampicillin, chloramphenicol, and tetracycline. However, all S. enterica serovars Enteritidis isolates were sensitive to all tested antimicrobial agents (Table 3).

Ten of 18 screened resistance associated genes were amplified in the S. enterica serovars Typhimurium isolates (Table 6). All isolates harbourd cat 1 associated with chloramphenicol resistance. While, 98.3, 96.6 and 94.8\% of S. enterica serovars Typhimurium isolates were possessed sul3 (sulphamethoxazole resistance), tetC (tetracycline resistance) and aadA2 (streptomycin resistance), respectively. Moreover, $65.5,84.5,56.9,62.1$ and $79.3 \%$ of $S$. enterica serovars Typhimurium were harboured ampicillin (BlaTEM), tetracycline (tetA), sulphamethoxazole (sul1), streptomycin (strA) and chloramphenicol (flo R) resistance associated genes, respectively (Table 6; Fig. 1c). Eight of the 18 screened resistance genes were amplified in the S. enterica serovars Enteritidis isolates; these were tetracycline resistance tet $\mathrm{A}(50 \%)$, and tetC (33.3\%); sulphamethoxazole resistance sul1 (16.7\%); streptomycin resistance aadA1 (50\%) and strA (33.3\%); chloramphenicol resistance cat 1 (33.3\%) and floR (16.7\%). The un-typable Salmonella isolates were only positive for two genes; $100 \%$ for tet $\mathrm{A}$ (tetracycline resistance) and $33.3 \%$ for cat 1 (chloramphenicol resistance). Only four of the screened genes tet $\mathrm{B}$ (tetracycline resistance), sul 2 (sulfamethoxazole resistance) and $a a d \mathrm{~B}$ and $a a c \mathrm{C}$ (gentamycin resistance) were not amplified in all screened isolates.

The amplicons of intI integrons were identified with size of $2 \mathrm{kbp}$ in three $S$. enteric serovar Typhimurium strains (Table 6). The sequencing data indicated that these integrons contain $d f r \mathrm{~A} 12$-orff-aadA2.

\section{Discussion}

Salmonella enterica serovars Typhimurium is known to be able to cause high rates of mortality in early ages of broiler chickens [20]. The InvA protein is a putative inner membrane component of the Salmonella pathogenicity island 1 (SPI-1) type 3 secretion system (TTSS) [40]. It has been reported that invA is present only in Salmonella species and therefore is used as a golden marker in genetic diagnosis of Salmonella species [35]. In this study 17 broiler flocks were positive and 67 Salmonella strains were isolated. The overall rate of incidence of Salmonella was (41\%) in the screened broiler chicken flocks which was considerably higher than the infection rates that reported in the UK (10.7\%), Lithuania (29\%), Italy (20\%), Netherlands (11\%) and Germany $(27.5 \%$ in chickens and $33.3 \%$ in turkeys) [41-45]. The higher infection rate found in this study compared to that of Abd El-Ghany et al. [18] shows the increased sensitivity of the use of the invA gene marker for diagnosis compared to isolation through culture on specific agar. 
Table 6 Prevalence of antibiotic resistant associated genes detected in S. enterica serovars

\begin{tabular}{|c|c|c|c|c|}
\hline \multirow[t]{2}{*}{ Resistance markers } & \multicolumn{3}{|c|}{ Prevalence of resistance genes in screened Salmonella isolates } & \multirow[t]{2}{*}{ Antimicrobial agent } \\
\hline & $\begin{array}{l}\text { S. Typhimurium } \\
\mathrm{n}=58\end{array}$ & $\begin{array}{l}\text { S. Enteritidis } \\
\mathrm{n}=6\end{array}$ & $\begin{array}{l}\text { Non-typable } \\
n=3\end{array}$ & \\
\hline BlaTEM & 38 & 0 & 0 & Ampicillin \\
\hline tetA & 49 & 3 & 3 & Tetracycline \\
\hline tet $B$ & 0 & 0 & 0 & Tetracycline \\
\hline tetC & 56 & 2 & 0 & Tetracycline \\
\hline sul1 & 33 & 1 & 0 & Sulphamethoxazole \\
\hline sul2 & 0 & 0 & 0 & Sulphamethoxazole \\
\hline sul3 & 57 & 0 & 0 & Sulphamethoxazole \\
\hline aadA1 & 24 & 3 & 0 & Streptomycin \\
\hline $\operatorname{aadA2}$ & 55 & 2 & 0 & Streptomycin \\
\hline strA & 36 & 2 & 0 & Streptomycin \\
\hline$s t r B$ & 0 & 0 & 0 & Streptomycin \\
\hline $\operatorname{aad} B$ & 0 & 0 & 0 & Gentamycin \\
\hline aacc & 0 & 0 & 0 & Gentamycin \\
\hline cat1 & 58 & 2 & 1 & Chloramphenicol \\
\hline cat2 & 0 & 0 & 0 & Chloramphenicol \\
\hline$f l o R$ & 46 & 1 & 0 & Chloramphenicol \\
\hline$d f r l$ & 0 & 0 & 0 & Trimethoprim \\
\hline intl & 3 & 0 & 0 & Class I integron \\
\hline
\end{tabular}

Although the S. enterica serovars Enteritidis is closely related to other pathogenic $S$. enteric serovars, this serovar has some characteristics that appear to discriminate it from others serovars. As S. enterica is known to contain the Salmonella difference fragments (sdf), a group of chromosomally encoded genes, which to date are of unknown function. $s d f$ I was reported by Agron et al. [33] to be found only in S. enterica serovars Enteritidis strains and considered to be a strong marker for this Salmonella serovar. $s d f$ I was used as a target for phylotying of the serotype-specific $S$. enterica serovars Enteritidis. In this study, $s d f$ l was present in 6 of the $67 i n v \mathrm{~A}$ positive isolates. These $s d f 1$ positive strains were isolated from three of 41 screened farms. Our findings indicated that the $s d f$ III gene marker was associated with the $s d f$ l positive strains. Interestingly, sdfII was detected in all 67 strains isolated in this study of different serovars. This indicates that there is some degree of diversity within serovars that can be detected by the primers which in agreement with previous observation $[33,35]$.

Bacteria use the fimbriae in the adherence to one another and to the host cells and in some instance to inanimate objects. Sef14 fimbriae have been shown to consist of a repeating major subunit of the $14.3 \mathrm{kDa}$ protein SefA, encoded for by the sefA gene and are required for macrophage uptake and survival in intraperitoneal infections [46]. The sefA gene is known to be specific to the poultry-associated Salmonella serotypes
Gallinarum and Enteritidis. It is also detected in serotype Dublin, although this serotype is more commonly associated with cattle [47]. In the present study, sefA was detected in all isolates of $S$. enterica serovar Enteritidis. In the current study same six $S$. enterica serovar Enteritidis isolates positive for $s d f \mathrm{f}$ and $s d f I I I$ markers were also positive for the sefA gene; these six strains came from three Salmonella infected chicken farms that were isolated from 41 screened farms.

In this study the $S$. enteric serovar Typhimurium serotype specific virulent flagella genes Typh and $f l i C$ were used for phenotyping as recommended previously [34, 35]. Flagella are multi-functional organelles that play different roles in the biology of bacteria. The motility functions of flagella help bacteria to acquire nutrients, move away from toxic materials, and move to specific colonization sites within hosts and to disperse in the environment during the course of transmission between hosts [48]. The flagellum also primes the host immune system through activation of TLR5 receptors [49].

sop $\mathrm{E}$ is a translocated effector protein that plays an important part in the systemic phase of salmonellosis infection; sop $\mathrm{E}$ has been shown to be involved in actin cytoskeletal rearrangements and membrane ruffling [36]. As a virulence factor that is frequently transferred by bacteriophages, the sop E gene is encoded in the SPI1 , and has been identified in isolates involved in major 
epidemics; sop $\mathrm{E}$ has therefore been identified as playing a key role in the emergence of epidemic strains [50].

In study conducted by Rahman et al. [51] indicated that sop $\mathrm{E}$ gene appeared to be distributed and conserved among only a few serovars of Salmonella (Enteritidis, Gallinarum and Virchow) irrespective of their source of isolation and the presence of sop E gene in Salmonella provides an important pathogenic means to invade epithelial cells [51]. Moreover Prager et al. [52] identified $s o p \mathrm{E}$ in all isolates of S. enterica serovar Enteritidis and carrying of sopE in $S$. Enteritidis may contribute to their epidemiological success [52]. In another study, all Salmonella Enteritidis isolated from human, chicken, and egg houses tested positive for $\operatorname{sop} E$ which may indicate its importance in pathogenesis [53].

In this study $92.5 \%$ of the Salmonella stains were harboured sopE gene that suggested that these strains could have zoonotic potential as previously reported [50-53].

There was a significant difference in mortality rate between Salmonella infected and non-infected flocks at the 1st week of life, however, there was no difference in mortality between Salmonella infected and non-infected flocks at the 5th week of age; a similar finding was previously reported [20,23]. According to previous study, the results suggest that the age at infection plays an important role in the persistence of $S$. enteritidis infection in chickens and may cause severe infections and high mortality in young chickens [54]. Unfortunately, in this study we did not investigate other possible causes of mortality which may act as co-factors.

There was a higher rate of Salmonella isolation from the sampled internal organs, in the gall bladder and liver samples compared to the intestine samples indicating the ability of Salmonella to cause systemic infection which in agreement with previous study [55].

In this study All S. enterica serovars Typhimurium and non-typable Salmonella strains isolated in this study were resistance to ampicillin, chloramphenicol, and tetracycline.

All isolates were sensitive to gentamicin. The susceptibility of S. enterica serovars Typhimurium to streptomycin and trimethoprim/sulphamethoxazole were 89.7 and $94.8 \%$, respectively. In addition $10.3 \%$ had intermediate sensitivity to streptomycin while all non-typable Salmonella strains were sensitive to trimethoprim/sulphamethoxazole and streptomycin. However, all S. enterica serovars Enteritidis isolates were sensitive to all tested antimicrobial agents. In contrast Salmonella isolates from South African chickens exhibited resistance to tetracycline $(93 \%)$, trimethoprim-sulfamethoxazole (84\%), gentamicin (48\%), ampicillin (47\%), chloramphenicol (31\%), and streptomycin (12\%) [56].
Most of the phenotypically antibiotic resistance isolates were positive for some of the antibiotic resistance marker genes for each of the screened antibiotics.

The blaTEM gene was detected only in $65.5 \%$ of ampicillin resistant $S$. enterica serovar Typhimurium isolates. All of the isolated strains of S. enterica serovar Enteritidis were susceptible to ampicillin and were negative for blaTEM. The three non-typable Salmonella strains showed phenotypical resistance to ampicillin without harbouring the blaTEM gene, indicating that these strains possess another ampicillin resistance mechanism.

In this study, tetracycline resistance in the S. enterica serovar Typhimurium isolates correlated with the presence of tetC (96.6\%), and tet $\mathrm{A}$ (84.5\%). All tested strains were negative for tet $\mathrm{B}$ codon. tet $\mathrm{A}$ codon was also found in all of the non-typable Salmonella strains. All S. enterica serovar Enteritidis were sensitive to tetracycline. However, two of the strains were harboured both tetC and tetA determinants and one strain was harbouring tetA determinant. These cassettes were silent in this serotype strain in vitro, however, they may turn on in vivo.

All of the S. enterica serovar Enteritidis and non-typable Salmonella strains were sensitive to trimethoprimsulphamethoxazole and all these strains were negative for the $d f r 1$ codon and did not possess integron that contains $d f r A 12$ trimethoprim resistance cassette. Although one strain of S. enterica serovar Enteritidis carried sul1 gene but not possessed any trimethoprim genes. All S. enterica serovar Typhimurium isolates were sensitive to trimethoprim-sulphamethoxazole despite $98 \%$ of isolates being positive for sul3 and $57 \%$ being positive for sul1, both of which confer sulphamethoxazole resistance. Interestingly, the three $S$. Typhimurium strains that were resistant to trimethoprim-sulphamethoxazole were found to harbour the $2 \mathrm{kp}$ integron that contains the $d f r A 12$ trimethoprim resistant marker.

All of the Salmonella isolates were sensitive to the streptomycin despite the presence of streptomycin modifying enzyme gene cassettes (aadA1, aadA2 and strA). This suggests that some of the antimicrobial resistance genes are silent in bacteria in vitro; however, these silent genes can spread to other bacteria or turn on in vivo, especially under antimicrobial pressure which in agreement with previous reports [31, 57].

The cat1 gene, encoding chloramphenicol acetyltransferase, was identified in all resistant strains. In $S$. enterica serovar Typhimurium, the cat 2 gene was not found in any of the tested strains. The floR gene which also confers chloramphenicol resistance was detected in $80 \%$ of $S$. enterica serovar Typhimurium strains. One of the non-typable Salmonella strains carried the cat 1 gene but the other two isolates did not possess cat 1 , cat 2 or floR gene indicating that these two strains 
harbour another chloramphenicol resistance mechanism. Of six S. enterica serovar Enteritidis strains, one strain possessed both, cat1 and, floR, and one strain harboured only the cat 1 gene, however, phenotypically they were all sensitive to chloramphenicol indicating that this resistant cassette is silent in vitro in this Salmonella serovar.

Multiple drug resistance genes have been found to be clustered on individual mobile elements, which mean that multi-resistance can be readily transferred and increase the multi-drug resistant bacterial population as reported previously [58].

Gene cassettes are a major source of the resistance genes found in clinical, commensal, and environmental isolates of bacteria that are resistant to antibiotics [59, $60]$. Most commonly, they are found in association with class 1 or class 2 integrons [61].

In this study, a class one integron in three S. enterica serovar Typhimurium strains with size of $2 \mathrm{~kb}$ was identified. The sequencing data indicated that these integrons contained $d f r \mathrm{~A} 12-o r f F-a a d \mathrm{~A} 2$. The presence of the $d f$ A12-orfF-aadA2 open reading frames revealed the basis for the streptomycin and trimethoprim/sulphamethoxazole resistance seen in these strains. It also provides an indication of the mapping distribution of antibiotic resistance alleles in this region of the Salmonella genome/chromosome.

In this study the higher infection rate in the investigated flocks may regarding to low biosecurity and hygienic measures inside these farms and easily to spread the infection through different reservoirs and the workers in the farms.

The screening of antimicrobial resistance in the Salmonella strains isolated in this study provides evidence for confirming the mechanisms employed by $S$. enterica serovars to resist cluster antibiotics used for treatment of broiler chicken in Egypt. Future work, in this regard, should address if allele distribution in chicken and human Salmonella isolates from the same region share the same resistance mechanisms in order to highlight potential horizontal gene transfer by this zoonotic organism and the origin of antimicrobial resistance in human isolates. Finally, we believe that this is the first report of the presence of a class one integron in the S. enterica serovar Typhimurium serotype together with the verification of the location of some resistance genes that are within or associated with the class one integron.

\section{Authors' contributions}

HES, AT, ME, TG and AAE participated in the conception and design of the study. HES, and AT were performed farm and laboratory work. HES, AT, ME, TG, $Y K, A A E, H N, H E$ and $H M H$ analysed the data and wrote the manuscript. FE participated in manuscript revision. All authors contributed to the analysis and supported the manuscript discussion. All authors read and approved the final manuscript.

\begin{abstract}
Author details
${ }^{1}$ Department of Chemistry and Biomolecular Science, Faculty of Engineering, Gifu University, 1-1 Yanagido, Gifu 501-1193, Japan. ${ }^{2}$ Institute of Bacterial Infections and Zoonoses, Friedrich-Loeffler-Institut, Naumburger Str. 96a, 07743 Jena, Germany. ${ }^{3}$ Faculty of Veterinary Medicine, Kafr Elsheikh University, Kafr El-Sheikh 33516, Egypt. ${ }^{4}$ CALM_live Imaging Facility, Centre for Inflammation Research, University of Edinburgh, Edinburgh 47 EH16 4TJ, UK. ${ }^{5}$ Institute of Poultry Diseases, Free University Berlin, Berlin, Germany.
\end{abstract}

\section{Acknowledgements}

We thank Professor Mark Steven and David Gally for their help to facilitate the conduction of the AST at the Clinical Veterinary Microbiology Laboratory of the Royal Dick School of Veterinary Study, University of Edinburgh.

\section{Competing interests}

The authors declare that they have no competing interests.

\section{Availability of data and materials}

The data supporting the findings of this study are contained within the manuscript.

\section{Ethics approval and consent for participate}

This study was carried out in strict accordance with the recommendations of the, Egyptian Network of Research Ethics Committees (ENREC) which complies with the international laws and regulation regarding ethical considerations in research.

\section{Funding}

The study was supported by Government of Republic of Egypt and the Faculty of Veterinary Medicine, Kafr El-shiekh University, and Department of Chemistry and Biomolecular science, Faculty of Engineering, Gifu University.

Received: 29 November 2016 Accepted: 3 February 2017

Published online: 10 February 2017

References

1. Hafez H. Salmonella infections in poultry: diagnosis and control. Period Biol. 2001;103:103-14

2. Thorns C. Bacterial food-borne zoonoses. Revue scientifique et technique. 2000;19:226-39.

3. Omwandho C, Kubota T. Salmonella enterica serovar Enteritidis: a minireview of contamination routes and limitations to effective control. Jpn Agric Res Q. 2010;44:7-16.

4. Bäumler A, Tsolis R, Ficht T, Adams L. Evolution of host adaptation in Salmonella enterica. Infect Immun. 1998;66:4579-87.

5. Popoff M, Bockemühl J, Brenner F. Supplement 1998 (no. 42) to the Kauffmann-White scheme. Res Microbiol. 2000;151:63-5.

6. Abouzeed $\mathrm{Y}$, Hariharan $\mathrm{H}$, Poppe C, Kibenge F. Characterization of Salmonella isolates from beef cattle, broiler chickens and human sources on Prince Edward Island. Comp Immunol Microbiol Infect Dis. 2000;23:253-66.

7. Sheela R, Babu U, Mu J, Elankumaran S, Bautista D, Raybourne R, et al. Immune responses against Salmonella enterica serovar enteritidis infection in virally immunosuppressed chickens. Clin Diag Lab Immunol. 2003;10:670-9.

8. Altekruse S, Bauer N, Chanlongbutra A, DeSagun R, Naugle A, Schlosser W, et al. Salmonella enteritidis in broiler chickens, United States, 20002005. Emerg Infect Dis. 2006;12:1848.

9. Coble D, Sandford E, Ji T, Abernathy J, Fleming D, Zhou H, et al. Impacts of Salmonella enteritidis infection on liver transcriptome in broilers. Genesis. 2013;51:357-64.

10. Gast R. Salmonella infections and Paratyphoid infections. In: Calnek BW, editor. Diseases of Poultry. Ames: lowa State University Press; 1997. p. 97-121.

11. Hafez H. Salmonella infections in Turkeys. In: Barrow P, Methner U, editors. Salmonella in domestic animals. Wallingford: CABI Publishing; 2013. p. 193-220 
12. Kapperud G, Stenwig H, Lassen J. Epidemiology of Salmonella typhimurium 0: 4-12 infection in Norway evidence of transmission from an avian wildlife reservoir. Am J Epidemiol. 1998;147:774-82.

13. OIE. Fowl typhoid and pullorum disease. In: Manual of standards for diagnostic tests and vaccines. 1996. p. 532-38.

14. Shivaprasad H, Chin R. Pullorum disease and fowl typhoid American Association of Avian Pathologists. 2001.

15. Majowicz S, Musto J, Scallan E, Angulo F, Kirk M, O'Brien S, et al. The global burden of nontyphoidal Salmonella gastroenteritis. Clin Infect Dis. 2010;50:882-9.

16. Newell D, Koopmans M, Verhoef L, Duizer E, Aidara-Kane A, Sprong $H$, et al. Food-borne diseases: the challenges of 20 years ago still persist while new ones continue to emerge. Int J Food Microbiol. 2010;139:S3-15.

17. Desmidt M, Ducatelle R, Haesebrouck F. Pathogenesis of Salmonella enteritidis phage type four after experimental infection of young chickens. Vet Microbiol. 1997;56:99-109.

18. Abd El-Ghany W, El-Shafii S, Hatem M. A survey on Salmonella species isolated from chicken flocks in Egypt. Asian J Anim Vet Adv. 2012;7:489-501.

19. Lister S. Salmonella enteritidis infection in broilers and broiler breeders. Vet Rec. 1988;123:350.

20. Padron M. Salmonella typhimurium outbreak in broiler chicken flocks in Mexico. Avian Dis. 1990;34:221-3.

21. Forsythe R, Ross W, Ayres J. Salmonellae recovery following gastrointestinal and ovarian inoculation in the domestic fowl. Poult Sci. 1967;46:849-55.

22. Hogue A, White P, Guard-Petter J, Schlosser W, Gast R, Ebel E, et al. Epidemiology and control of egg-associated Salmonella Enteritidis in the United States of America [Review]. Rev Sci Tech. 1997;16:542-53.

23. Barbour E, Jurdi L, Talhouk R, Qatanani M, Eid A, Sakr W, et al. Emergence of Salmonella enteritidis outbreaks in broiler chickens in the Lebanon: epidemiological markers and competitive exclusion control. Revue scientifique et technique. 1999:18:710-8.

24. Barrow P. Experimental infection of chickens with Salmonella enteritidis. Avian Pathol. 1991;20:145-53.

25. Rampling A, Upson R, Ward L, Anderson J, Peters E, Rowe B. Salmonella enteritidis phage type 4 infection of broiler chickens: a hazard to public health. Lancet. 1989;334:436-8.

26. Su L, Chiu C, Chu C, Ou J. Antimicrobial resistance in nontyphoid Salmonella serotypes: a global challenge. Clin Infect Dis. 2004;39:546-51.

27. Chen S, Zhao S, White D, Schroeder C, Lu R, Yang H, et al. Characterization of multiple-antimicrobial-resistant Salmonella serovars isolated from retail meats. Appl Environ Microbiol. 2004;70:1-7.

28. Lynne A, Rhodes-Clark B, Bliven K, Zhao S, Foley S. Antimicrobial resistance genes associated with Salmonella enterica serovar Newport isolates from food animals. Antimicrob Agents Chemother. 2008:52:353-6.

29. Blair J, Webber M, Baylay A, Ogbolu D, Piddock L. Molecular mechanisms of antibiotic resistance. Nat Rev Microbiol. 2015;13:42-51.

30. Okeke I, Laxminarayan R, Bhutta Z, Duse A, Jenkins P, O'Brien T, et al. Antimicrobial resistance in developing countries. Part l: recent trends and current status. Lancet Infect Dis. 2005;5:481-93.

31. Ma M, Wang H, Yu Y, Zhang D, Liu S. Detection of antimicrobial resistance genes of pathogenic Salmonella from swine with DNA microarray. J Vet Diagn Investig. 2007;19:161-7

32. Malorny B, Bunge C, Helmuth R. Discrimination of d-tartrate-fermenting and-nonfermenting Salmonella enterica subsp. enterica isolates by genotypic and phenotypic methods. J Clin Microbiol. 2003:41:4292-7.

33. Agron P, Walker R, Kinde H, Sawyer S, Hayes D, Wollard J, et al. Identification by subtractive hybridization of sequences specific for Salmonella enterica serovar Enteritidis. Appl Environ Microbiol. 2001;67:4984-91.

34. Alvarez J, Sota M, Vivanco A, Perales I, Cisterna R, Rementeria A, et al. Development of a multiplex PCR technique for detection and epidemiological typing of Salmonella in human clinical samples. J Clin Microbiol. 2004;42:1734-8

35. O'Regan E, McCabe E, Burgess C, McGuinness S, Barry T, Duffy G, et al. Development of a real-time multiplex PCR assay for the detection of multiple Salmonella serotypes in chicken samples. BMC Microbiol. 2008;8:1.

36. Prager R, Rabsch W, Streckel W, Voigt W, Tietze E, Tschäpe H. Molecular properties of Salmonella enterica serotype paratyphi B distinguish between its systemic and its enteric pathovars. J Clin Microbiol. 2003:41:4270-8.
37. Bauer A, Kirby W, Sherris J, Turck M. Antibiotic susceptibility testing by a standardized single disk method. Am J Clin Pathol. 1966;45:493.

38. EUCAST. Breakpoint tables for interpretation of MICs and zone diameters. ed.: version 2015

39. Guard-Petter J, Parker C, Asokan K, Carlson R. Clinical and veterinary isolates of Salmonella enterica serovar Enteritidis defective in lipopolysaccharide O-chain polymerization. Appl Environ Microbiol. 1999;65:2195-201.

40. Shah D, Zhou X, Addwebi T, Davis M, Orfe L, Call D, et al. Cell invasion of poultry-associated Salmonella enterica serovar Enteritidis isolates is associated with pathogenicity, motility and proteins secreted by the type III secretion system. Microbiology. 2011;157:1428-45.

41. Hafez H, Stadler A, Kosters J. Surveillance on Salmonella in turkey flocks and processing plants. Dtsch Tierarztl Wochenschr. 1997;104:33-5.

42. Pieskus J, Franciosini M, Proietti PC, Reich F, Kazeniauskas E, ButrimaiteAmbrozeviciene C, et al. Preliminary investigations on Salmonella spp. incidence in meat chicken farms in Italy, Germany, Lithuania and the Netherlands. Int J Poult Sci. 2008;7:813-7.

43. Snow L, Davies R, Christiansen K, Carrique-Mas J, Cook A, Teale C, et al. Survey of the prevalence of Salmonella on commercial broiler farms in the United Kingdom, 2005/06. Vet Rec. 2008;163:649-54.

44. Van Hoorebeke S, Van Immerseel F, Schulz J, Hartung J, Harisberger M, Barco $L$, et al. Determination of the within and between flock prevalence and identification of risk factors for Salmonella infections in laying hen flocks housed in conventional and alternative systems [Research Support Non-U.S. Gov't]. Prev Vet Med. 2010:94:94-100.

45. Van Overbeke I, Duchateau L, De Zutter L, Albers G, Ducatelle R. A comparison survey of organic and conventional broiler chickens for infectious agents affecting health and food safety. Avian Dis. 2006;50(2):196-200.

46. Edwards R, Schifferli D, Maloy S. A role for Salmonella fimbriae in intraperitoneal infections. Proc Natl Acad Sci. 2000;97:1258-62.

47. Clayton D, Bowen A, Hulme S, Buckley A, Deacon V, Thomson N, et al. Analysis of the role of 13 major fimbrial subunits in colonisation of the chicken intestines by Salmonella enterica serovar Enteritidis reveals a role for a novel locus. BMC Microbiol. 2008;8:1.

48. Girón J. Role of flagella in mucosal colonization. Colonization of mucosal surfaces. Boston: American Society of Microbiology; 2005. p. 213-36.

49. Tahoun A, Jensen K, Corripio-Miyar Y, McAteer S, Corbishley A, Mahajan $A$, et al. Functional analysis of bovine TLR5 and association with $\lg A$ responses of cattle following systemic immunisation with $\mathrm{H} 7$ flagella. Vet Res. 2015;46:1.

50. Hopkins K, Threlfall E. Frequency and polymorphism of sopE in isolates of Salmonella enterica belonging to the ten most prevalent serotypes in England and Wales. J Med Microbiol. 2004;53:539-43.

51. Rahman H, Streckel W, Prager R, Tschape H. Presence of sopE gene and its phenotypic expression among different serovars of Salmonella isolated from man and animals. Indian J Med Res. 2004;120:35-8.

52. Prager R, Mirold S, Tietze E, Strutz U, Knuppel B, Rabsch W, et al. Prevalence and polymorphism of genes encoding translocated effector proteins among clinical isolates of Salmonella enterica. Int J Med Microbiol. 2000;290:605-17.

53. Han J, Gokulan K, Barnette D, Khare S, Rooney A, Deck J, et al. Evaluation of virulence and antimicrobial resistance in Salmonella enterica serovar Enteritidis isolates from humans and chicken- and egg-associated sources. Foodborne Pathog Dis. 2013;10:1008-15.

54. Gorham S, Kadavil K, Lambert H, Vaughan E, Pert B, Abel J. Persistence of Salmonella enteritidis in young chickens. Avian Pathol. 1991;20:433-7.

55. Bolder N, Janss L, Putirulan F, Wagenaar J. Resistance of broiler outbred lines to infection with Salmonella enteritidis. Avian Pathol. 2002;31:581-7.

56. Zishiri O, Mkhize N, Mukaratirwa S. Prevalence of virulence and antimicrobial resistance genes in Salmonella spp. isolated from commercial chickens and human clinical isolates from South Africa and Brazil. Onderstepoort J Vet Res. 2016;83:e1-11.

57. Maynard C, Fairbrother J, Bekal S, Sanschagrin F, Levesque R, Brousseau $\mathrm{R}$, et al. Antimicrobial resistance genes in enterotoxigenic Escherichia coli O149: K91 isolates obtained over a 23-year period from pigs. Antimicrob Agents Chemother. 2003;47:3214-21.

58. Nikaido H. Multidrug resistance in bacteria. Annu Rev Biochem. 2009;78:119.

59. Walsh T. Combinatorial genetic evolution of multiresistance. Curr Opin Microbiol. 2006:9:476-82 
60. Yang H, Byelashov O, Geornaras I, Goodridge L, Nightingale K, Belk K, et al. Presence of antibiotic-resistant commensal bacteria in samples from agricultural, city, and national park environments evaluated by standard culture and real-time PCR methods. Can J Microbiol. 2010;56:761-70.

61. Dawes F, Kuzevski A, Bettelheim K, Hornitzky M, Djordjevic S, Walker M. Distribution of class 1 integrons with IS 26-mediated deletions in their $3^{\prime}$-conserved segments in Escherichia coli of human and animal origin. PLOS ONE. 2010;5:e12754.

62. Randall L, Cooles S, Osborn M, Piddock L, Woodward M. Antibiotic resistance genes, integrons and multiple antibiotic resistance in thirty-five serotypes of Salmonella enterica isolated from humans and animals in the UK. J Antimicrob Chemother. 2004;53:208-16.
63. Boerlin P, Travis R, Gyles C, Reid-Smith R, Lim N, Nicholson V, et al. Antimicrobial resistance and virulence genes of Escherichia coli isolates from swine in Ontario. Appl Environ Microbiol. 2005;71:6753-61.

64. Fonseca E, Mykytczuk O, Asensi M, Reis E, Ferraz L, Paula F, et al. Clonality and antimicrobial resistance gene profiles of multidrug-resistant Salmonella enterica serovar infantis isolates from four public hospitals in Rio de Janeiro, Brazil. J Clin Microbiol. 2006;44:2767-72.

65. Zhao S, White D, Ge B, Ayers S, Friedman S, English L, et al. Identification and characterization of integron-mediated antibiotic resistance among Shiga toxin-producing Escherichia coli isolates. Appl Environ Microbiol. 2001;67:1558-64

\section{Submit your next manuscript to BioMed Central and we will help you at every step:}

- We accept pre-submission inquiries

- Our selector tool helps you to find the most relevant journal

- We provide round the clock customer support

- Convenient online submission

- Thorough peer review

- Inclusion in PubMed and all major indexing services

- Maximum visibility for your research

Submit your manuscript at www.biomedcentral com/submit 